\title{
Riesgo de diabetes y prediabetes en Manizales, estudio RIDIMA
}

\author{
Diabetes and prediabetes risk in Manizales, RIDIMA study
}

\author{
Germán Camilo Giraldo González¹, Santiago José Morón Serrano², Daniel Fernando Giraldo \\ Ceballos', María de los Ángeles Araque Coronado', Germán Camilo Duque Gallego², Nicolás \\ Esteban Torres Riascos ${ }^{2}$, Juanita González Escobar ${ }^{2}$
}

${ }^{1}$ Médico Internista Universidad de Caldas, Fellow de Cardiología Universidad ICESI Fundación Valle del Lili.

${ }^{2}$ Estudiante de Medicina Universidad de Caldas.

Correspondencia: german.camilo.giraldo@gmail.com.

Fecha de recepción: 20/10/2018

Fecha de aceptación: 28/11/2018

\section{Resumen}

Introducción: La diabetes mellitus es una enfermedad crónica, no transmisible, cuya prevalencia ha ido en aumento en los últimos años, convirtiéndose en una pandemia y un problema de salud pública que requiere intervenciones desde la prevención y el diagnóstico temprano para poder evitar la aparición tanto de la enfermedad como de las múltiples complicaciones que acarrea. Dentro de los intentos por encontrar herramientas de tamización que permitan cumplir este objetivo está el FINDRISC, un cuestionario que estima el riesgo de padecer diabetes a futuro y ha sido validado en Colombia, asignando a la población de riesgo un puntaje mayor o igual a 13.

Objetivo: Detectar, en adultos de Manizales, el riesgo de presentar diabetes mellitus tipo 2, usando la herramienta FINDRISC y realizar una descripción de esta misma población en términos de peso, índice de masa corporal (IMC), circunferencia abdominal, antecedentes familiares de diabetes, estado socioeconómico y nivel educativo.

Metodología: Estudio de corte transversal, en el cual se aplicó el cuestionario FINDRISC a adultos de la ciudad de Manizales que no tuviesen diagnóstico de diabetes y a aquellos con puntaje de riesgo ( $\geq$ a 13 puntos) se les realizó una prueba de glucometría ocasional y se sugirió asistir a consulta médica para ampliar los estudios. Los cuestionarios se realizaron entre febrero y noviembre de 2017.

Resultados: Participaron 1.000 personas con edad promedio de 43 años $( \pm 16)$. La prevalencia de participantes con puntaje FINDRISC $\geq 13$ fue del 14,4\% y no se halló relación entre el puntaje de riesgo y el valor de glucemia ocasional; el 31,9\% de las personas con puntaje de riesgo refieren que acataron la recomendación de acudir a consulta y realizarse una glucemia en ayunas, pero solo se obtuvo el resultado del $16,7 \%$ de los participantes en riesgo; de estos últimos, el 37,5\% tiene resultado de glucemia alterada en ayunas y el $8,3 \%$ de diabetes. El $42,7 \%$ de la población encuestada se encontraba en sobrepeso y el 12,5\% en algún grado de obesidad; el 25,6\% de los encuestados tuvieron perímetro abdominal aumentado.

Conclusiones: Manizales comparte la prevalencia descrita a nivel nacional de pacientes en riesgo para diabetes/prediabetes. Llamativamente, pocos pacientes acogieron la recomendación de asistir a un centro asistencial para ahondar en su riesgo. Los datos de sobrepeso y obesidad, acordes al país, siguen siendo elevados.

Palabras clave: Diabetes Mellitus Tipo 2, factores de riesgo, estado prediabético, índice de masa corporal, circunferencia abdominal, medición de riesgo.

\section{Abstract}

Introduction: Diabetes mellitus is a chronic, non-transmissible disease with a growing prevalence as measured in the last years, which turns diabetes into a public health problem and raises the need for interventions from prevention and early diagnosis aiming that the appearance and complications of this disease can be avoided. FINDRISC is a questionnaire used as a screening tool to measure the risk of developing diabetes in the future. This questionnaire has been validated in Colombia, where a threshold of 13 or more points is accepted as risk score.

Aims: The aim is to detect the risk of developing Diabetes Mellitus 2 in the adult population of Manizales by using the FINDRISC questionnaire. Also it is pretended to describe this population in terms of weight, body mass index (BMI), waist circumference, family history of diabetes, socioeconomic status and educational level. 
Methods: It is an analytic study in which FINDRISC questionnaire was applied to adults from Manizales that hadn't been diagnosed with diabetes. Those with a score equal to or higher than 13 underwent random plasma glucose test and were given the indication of consulting a doctor and undergo fasting plasma glucose test. The questionnaires were made in 2017, between February and November.

Results: There were 1000 people in the study; the average age was 43 years $( \pm 16)$. The prevalence of FINDRISC equal to or higher than 13 was $14.4 \%$ and there was no relation found between risk score and random plasma glucose. $31.9 \%$ of people with risk score reported that they had gone to the doctor and underwent the fasting glucose test; however only $16.7 \%$ of the risk scores' results were obtained. Of those, 37.5\% had fasting glucose impairment and $8.3 \%$ had a diabetes diagnosis. $42.7 \%$ of the whole population in the study was classified as overweight and $12.5 \%$ were classified as obesity. $25.6 \%$ of the population had an increased abdominal perimeter.

Conclusions: Manizales has a similar prevalence of diabetes/prediabetes compared to national prevalence. It is striking that only a few patients complied with the recommendation to find a health care center for an accurate diagnosis. Data on overweight and obesity are high, as they are in the Country.

Key words: Diabetes Mellitus, Type 2, risk Factors, prediabetic state, body mass index, abdominal circumference, risk assessment.

\section{Introducción}

La diabetes mellitus es una entidad crónica no transmisible, causante de desórdenes metabólicos, principalmente hiperglucemia crónica. Se produce como resultado de la interacción entre predisposición genética y factores de riesgo conductuales y medioambientales (obesidad, sedentarismo, antecedentes familiares, nutrición inadecuada, antecedente de alteración en la glucemia, etc.) y está asociada con lesiones macrovasculares y microvasculares causantes de las mayores complicaciones de la enfermedad ${ }^{(1,2,3,4)}$.

La prevalencia de la enfermedad ha ido aumentando en todo el mundo, convirtiéndose en un problema de salud pública y en una fuente importante de morbilidad y mortalidad. Para el año 2015, aproximadamente 415 millones de personas tenían diabetes mellitus y más de 300 millones de individuos tenían manifestaciones que indican riesgo de desarrollar diabetes mellitus tipo 2 en el futuro (prediabetes), adicionalmente se estima que para el año 2040 habrá 642 millones de personas diabéticas en el mundo ${ }^{(5,6)}$. En Colombia, en el 2016 se estimó que la prevalencia estaba entre $7 \%$ y $9 \%$ de la población adulta y se espera que para el año 2035 haya en total 3,34 millones de personas con la enfermedad. La diabetes mellitus tipo 2 no causa síntomas específicos, lo que explica que del $25 \%$ al $50 \%$ de los casos de la enfermedad permanecen sin diagnóstico ${ }^{(6,7,8)}$. Otras fuentes ${ }^{(9,10)}$ indican que en el 2013 en Colombia había 634.098 personas afiliadas al Sistema General de Seguridad Social en Salud con diagnóstico de diabetes mellitus donde se estima que el 5,1\% de mayores de 45 años tenía diabetes mellitus.

La herramienta FINDRISC (Finnish Diabetes Risk Score) se emplea como un método sencillo y no invasivo, útil en la identificación de las personas en riesgo de padecer diabetes mediante la evaluación de factores de riesgo asociados a la enfermedad y está en la guía de práctica nacional de esta enfermedad ${ }^{(11,12,13)}$. Se recomienda su uso y la realización de pruebas diagnósticas si hay puntaje mayor o igual a 13 puntos ${ }^{(8,14,15)}$; este puntaje fue el utilizado para el presente estudio. El objetivo de este estudio es identificar el riesgo de presentar diabetes/prediabetes con esta herramienta en adultos de la ciudad de Manizales.

\section{Materiales y métodos}

Previa presentación del proyecto y aprobación por parte del comité de ética de la Universidad de Caldas, se dispuso el grupo de investigadores a recolectar la información. Los criterios de inclusión eran: tener una edad $\geq 18$ años, ser residente de la ciudad de Manizales, firmar el consentimiento informado y se excluyó aquellos que ya tuvieran diagnóstico de diabetes mellitus. Con una prevalencia estimada en Colombia del 7\%, el tamaño de la muestra se calculó para una potencia del $80 \%$ con un estimado de sensibilidad de $90 \%$ y especificidad de $80 \%$ para detectar personas en riesgo de tener diabetes mellitus tipo 2, con un nivel de confianza de 95\% para un riesgo de error alfa de 3,1\% y una población $\geq 18$ de años en Manizales de 299.879 personas, se calculó una muestra de 997 participantes. La muestra se estratificó por conveniencia, distribuyendo el sitio de encuestas en zonas estratégicas que incluyeran todos los estratos socioeconómicos y zonas rurales y urbanas pertenecientes a la ciudad. Entre febrero y noviembre de 2017 se realizaron todas las entrevistas y se aplicaron las encuestas. Para los elementos médicos, sociodemográficos y factores de riesgo se realizó entrevista personal, para el peso y la talla se solicitó retirar los zapatos, y retirar los elementos de los bolsillos y dejar la ropa lo más liviana posible, no tener intención de micción o haberla presentado antes de las mismas. El índice de masa corporal se calculó como el peso dividido entre la talla en metros al cuadrado. La medida de la circunferencia se realizó en el punto medio entre la última costilla y la cresta ilíaca y se registró en centímetros. A todos los participantes que tuvieran un puntaje mayor o igual a 13 se les invitó a realizar una glucometría ocasional (al finalizar la entrevista) y se les entregó un documento firmado por los investigadores en el que se invitaba a solicitar una cita médica, se explicaba al médico tratante (o EPS aseguradora del paciente) el resultado del puntaje y la recomendación de la guía de ampliar las pruebas diagnósticas de diabetes, para tener, seis 
meses después, un nuevo contacto telefónico con estas personas en riesgo e indagar si habían acudido al médico, y el resultado de las pruebas realizadas. La IPS Médicos Internistas de Caldas aportó los equipos e insumos para la evaluación de los participantes. No tuvo participación en el diseño, análisis ni redacción del manuscrito.

\section{Análisis estadístico}

Para el análisis de la información se utilizó SPSS V.24.0 (Licenciado para la Universidad de Caldas) para el manejo de datos y análisis estadísticos. Las variables continuas se probaron para la normalidad de la distribución y se presentaron como media $\pm \mathrm{DE}$, y las variables categóricas como porcentajes. La comparación entre grupos se realizó utilizando la prueba $t$ de Student o la prueba $\chi 2$, según corresponda para el manejo de datos.

\section{Resultados}

En el estudio se incluyeron 1.000 participantes. La edad promedio fue de 43 ( \pm 16 años); el promedio de IMC fue de $25,7 \mathrm{~kg} / \mathrm{m}^{2}( \pm 4,1)$, el $42,7 \%$ tenía sobrepeso y el $12,5 \%$, obesidad. El 22,2\% de las mujeres encuestadas tuvo un perímetro abdominal mayor o igual a $90 \mathrm{~cm}$ con un promedio de $82,4 \mathrm{~cm}$ $( \pm 10,4)$, y el $28,3 \%$ de los hombres tuvo un perímetro mayor o igual a $94 \mathrm{~cm}$ con un promedio de $88,5 \mathrm{~cm}( \pm 10,6)$. El $45,7 \%$ de la muestra tenía algún antecedente familiar de diabetes mellitus tipo 2. Con respecto al nivel educativo, en la población encuestada el 22,3\% no había completado la educación básica, en contraste con el 77,7\% que había terminado el bachillerato y de éstos la mitad tenían estudios de educación superior; el $56,8 \%$ de la población encuestada se concentraba en los estratos 3 y 4 . Las variables demográficas y elemento incluidos en la escala "FINDRISC" se encuentran en la tabla 1.

\section{Participantes en riesgo}

De la población encuestada, $144(14,4 \%)$ personas obtuvieron un puntaje de riesgo (mayor o igual a 13 puntos), de los cuales el 41,7\% son mujeres. Al 100\% de estas personas se les realizó al instante una glucometría ocasional con una media de $108 \mathrm{mg} / \mathrm{dl}( \pm 24,5)$ y se encontró una persona con resultado de $271 \mathrm{mg} / \mathrm{dl}$ la cual se derivó al servicio médico para recibir la atención respectiva. No se halló correlación estadísticamente significativa entre el valor de la glucemia ocasional y el puntaje de riesgo obtenido.

De las personas con puntaje $\geq 13$, el $91,7 \%$ estaba en sobrepeso/obesidad, relación inversa en los participantes con puntaje $<13$, siendo este factor el que más puntaje otorgara para la clasificación de riesgo; el 81,9\% de personas en riesgo tenía un perímetro abdominal mayor al punto de corte. En cuanto a los estilos de vida saludable, se encontró que, de las personas con puntaje $\geq 13$, el 79,9\% no realizaba el ejercicio regular y el $66,7 \%$ no comía frutas y verduras todos los días; sin embargo,
Tabla 1. Variables demográficas y elementos incluidos en la escala FINDRISC

\begin{tabular}{|c|c|c|c|}
\hline Variable & $\begin{array}{l}\text { Mujeres n } \\
\text { (\% grupo) }\end{array}$ & $\begin{array}{l}\text { Hombres n } \\
\text { (\% grupo) }\end{array}$ & $\begin{array}{c}\text { Total n } \\
\text { (\% total) }\end{array}$ \\
\hline \multicolumn{4}{|l|}{ Edad años } \\
\hline $\begin{array}{l}<45 \\
45-54 \\
>64 \\
\text { Total }\end{array}$ & $\begin{array}{c}236(53,4) \\
182(41,2) \\
24(5,4) \\
442(100)\end{array}$ & $\begin{array}{l}283(50,7) \\
205(36,7) \\
70(12,5) \\
558(100)\end{array}$ & $\begin{array}{c}519(51,9) \\
387(38,7) \\
94(9,4) \\
1000(100)\end{array}$ \\
\hline \multicolumn{4}{|l|}{ IMC } \\
\hline $\begin{array}{l}<25 \\
25-30 \\
>30 \\
\text { Total }\end{array}$ & $\begin{array}{l}187(42,3) \\
191(43,2) \\
64(14,5) \\
442(100)\end{array}$ & $\begin{array}{c}261(46,8) \\
236(42,3) \\
61(10,9) \\
558(100)\end{array}$ & $\begin{array}{l}448(44,8) \\
427(42,7) \\
125(12,5) \\
1000(100)\end{array}$ \\
\hline \multicolumn{4}{|c|}{ Perímetro abdominal (centímetros) } \\
\hline $\begin{array}{l}\text { M: }<90 \mathrm{H}:<94 \\
M: \geq 90 \mathrm{H}: \geq 94 \\
\text { Total }\end{array}$ & $\begin{array}{c}344(77,8) \\
98(22,2) \\
442(100)\end{array}$ & $\begin{array}{l}400(71,7) \\
158(28,3) \\
558(100)\end{array}$ & $\begin{array}{l}744(74,4) \\
256(25,6) \\
1000(100)\end{array}$ \\
\hline \multicolumn{4}{|c|}{ Ejercicio $\mathbf{3 0}$ minutos diarios } \\
\hline $\begin{array}{l}\text { Sí } \\
\text { No } \\
\text { Total }\end{array}$ & $\begin{array}{c}96(21,7) \\
346(78,3) \\
442(100)\end{array}$ & $\begin{array}{l}170(30,5) \\
388(69,5) \\
558(100)\end{array}$ & $\begin{array}{l}266(26,6) \\
734(73,4) \\
1000(100)\end{array}$ \\
\hline \multicolumn{4}{|l|}{ Frutas/verduras } \\
\hline $\begin{array}{l}\text { Todos los días } \\
\text { No todos los días } \\
\text { Total }\end{array}$ & $\begin{array}{l}227(48,6) \\
215(51,4) \\
442(100)\end{array}$ & $\begin{array}{l}244(43,7) \\
314(56,3) \\
558(100)\end{array}$ & $\begin{array}{l}471(47,1) \\
529(52,9) \\
1000(100)\end{array}$ \\
\hline \multicolumn{4}{|l|}{ Medicación para HTA } \\
\hline $\begin{array}{l}\text { Sí } \\
\text { No } \\
\text { Total }\end{array}$ & $\begin{array}{c}50(11,3) \\
392(88,7) \\
442(100)\end{array}$ & $\begin{array}{c}82(14,7) \\
476(85,3) \\
558(100)\end{array}$ & $\begin{array}{l}132(13,2) \\
868(86,8) \\
1000(100)\end{array}$ \\
\hline \multicolumn{4}{|c|}{ Valores previos de glucemia alta } \\
\hline $\begin{array}{l}\text { Sí } \\
\text { No } \\
\text { Total }\end{array}$ & $\begin{array}{c}33(7,5) \\
409(92,5) \\
442(100)\end{array}$ & $\begin{array}{c}43(7,7) \\
515(92,3) \\
558(100)\end{array}$ & $\begin{array}{c}76(7,6) \\
924(92,4) \\
1000(100)\end{array}$ \\
\hline \multicolumn{4}{|c|}{ Historia familiar de diabetes } \\
\hline $\begin{array}{l}\text { Sí: } 1 \text { er o } 2 \text { do grado } \\
\text { No } \\
\text { Total }\end{array}$ & $\begin{array}{l}224(50,7) \\
218(49,3) \\
442(100)\end{array}$ & $\begin{array}{l}233(41,8) \\
325(58,2) \\
558(100)\end{array}$ & $\begin{array}{l}457(45,7) \\
543(54,3) \\
1000(100)\end{array}$ \\
\hline \multicolumn{4}{|l|}{ Nivel educativo } \\
\hline $\begin{array}{l}\text { Ninguno } \\
\text { Escuela incompleta } \\
\text { Escuela completa } \\
\text { Educación superior } \\
\text { Total }\end{array}$ & $\begin{array}{c}2(0,5) \\
80(4,5) \\
165(37,3) \\
195(16,3) \\
442(100)\end{array}$ & $\begin{array}{c}5(0,9) \\
136(5,7) \\
223(40) \\
194(15,2) \\
558(100)\end{array}$ & $\begin{array}{c}7(0,7) \\
216(21,6) \\
388(38,8) \\
389(38,9) \\
1000(100)\end{array}$ \\
\hline \multicolumn{4}{|l|}{ Estrato } \\
\hline $\begin{array}{l}1 \text { y } 2 \\
3 \text { y } 4 \\
5 \text { y } 6 \\
\text { Total }\end{array}$ & $\begin{array}{c}144(32,6) \\
253(57,2) \\
45(10,2) \\
442(100)\end{array}$ & $\begin{array}{c}194(34,8) \\
315(56,5) \\
49(8,8) \\
558(100)\end{array}$ & $\begin{array}{c}338(33,8) \\
568(56,8) \\
94(9,4) \\
1000(100)\end{array}$ \\
\hline \multicolumn{4}{|l|}{ Régimen de salud } \\
\hline $\begin{array}{l}\text { Subsidiado } \\
\text { Contributivo } \\
\text { Total }\end{array}$ & $\begin{array}{c}81(18,3) \\
361(81,7) \\
442(100)\end{array}$ & $\begin{array}{c}98(17,6) \\
460(82,4) \\
558(100)\end{array}$ & $\begin{array}{l}179(17,9) \\
821(82,1) \\
1000(100)\end{array}$ \\
\hline \multicolumn{4}{|l|}{ Puntaje FINDRISC } \\
\hline $\begin{array}{l}<13 \\
\geq 13 \\
\text { Total }\end{array}$ & $\begin{array}{c}382(86,4) \\
60(13,6) \\
442(100)\end{array}$ & $\begin{array}{c}474(84,9) \\
84(15,1) \\
558(100)\end{array}$ & $\begin{array}{r}856(85,6) \\
144(14,4) \\
1000(100)\end{array}$ \\
\hline
\end{tabular}

IMC: Índice de masa corporal H: Hombres M: Mujeres HTA: Hipertensión arterial 
en ambos grupos (con o sin riesgo) la mayoría de participantes no tenía estilos de vida saludables.

El 39,6\% de las personas con puntaje $\geq 13$ tenía antecedente de hipertensión arterial y tenía tratamiento farmacológico. De los participantes en riesgo, 53 $(36,8 \%)$ habían tenido valores previos de glucemia alta y $199(82,6 \%)$ tenían historia de diabetes en familiares de primer o segundo grado. La tabla 2 muestra las variables estudiadas en relación con el puntaje obtenido en el cuestionario.

En la llamada de seguimiento seis meses después de la evaluación, el 31,9\%(46) de los participantes en riesgo manifestó acudir a los exámenes de evaluación diagnóstica para DM2. Para el estudio, estuvo disponible el resultado de 24 . Las otras 98 personas en riesgo $(68,1 \%)$ no se realizaron los estudios, las razones fueron falta de interés u olvido $(91,8 \%)$ y problemas para obtener acceso a la atención (8,2\%). En la tabla 3 se sintetizan los resultados de las glucemias en ayunas obtenidas de los pacientes que se hicieron los estudios.

\section{Discusión}

La diabetes es una enfermedad asintomática de múltiples factores de riesgo ${ }^{(16)}$; normalmente pasa desapercibida, lo que hace que pasen años antes de su diagnósti$\mathrm{co}^{(6)}$. Se encontró un $14,4 \%$ de personas en riesgo y en el test de glucemia ocasional no hubo ninguna relación significativa aunque se encontró una persona con posible diagnóstico de diabetes. Comparativamente, Barengo y cols., en Barranquilla, en el estudio DEMOJUAN, aplicaron el cuestionario a 14.193 personas con características diferentes a las de este estudio en términos de género y edad ( $70 \%$ mujeres y $30 \%$ hombres con medias de edad de 48 y 50 años, respectivamente, versus 43 años promedio en este estudio y una mayoría masculina) donde el $35 \%$ obtuvo un puntaje $\geq 13^{(17)}$.

El porcentaje de personas en riesgo de desarrollar diabetes para la región de Suramérica y Centroamérica estuvo acorde a la descrita (14\%) con un $7,9 \%$ en el 2015 según la Federación Internacional de diabetes y en Colombia se estimaba entre el 7\% y $9 \%$, recordando que la mitad de estos continúan sin diagnóstico y otra mitad cursa con prediabetes ${ }^{(6,8)}$. Por otra parte, el estudio PURE-Colombia, con adultos entre 35 y 70 años, reportó una prevalencia de prediabetes del 11,9\%, ligeramente inferior a la hallada en Manizales por la escala $^{(18)}$.

La prevalencia de sobrepeso y obesidad correspondió al 55,2\% de los participantes, lo que concuerda con los porcentajes de sobrepeso y obesidad en el país que se calculan en más del $50 \%{ }^{(18)}$. Este comportamiento
Tabla 2. Variables estudiadas y su relación con el puntaje obtenido en el cuestionario

\begin{tabular}{|c|c|c|c|}
\hline Variable & $\begin{array}{c}\text { FINDRISC > } 13 \text { n } \\
\text { (\% grupo) }\end{array}$ & $\begin{array}{c}\text { FINDRISC < } 13 \text { n } \\
(\% \text { grupo })\end{array}$ & $\begin{array}{c}\text { Total } n \\
\text { (\% total) }\end{array}$ \\
\hline \multicolumn{4}{|l|}{ Edad } \\
\hline $\begin{array}{l}<45 \\
45-54 \\
55-64 \\
>64 \\
\text { Total }\end{array}$ & $\begin{array}{c}36(25) \\
45(31,3) \\
39(27,1) \\
24(16,6) \\
144(100)\end{array}$ & $\begin{array}{c}483(56,4) \\
171(20) \\
132(15,4) \\
70(8,2) \\
856(100)\end{array}$ & $\begin{array}{c}519(51,9) \\
216(21,6) \\
171(17,1) \\
94(9,4) \\
1000(100)\end{array}$ \\
\hline \multicolumn{4}{|l|}{ Género } \\
\hline $\begin{array}{l}\text { Masculino } \\
\text { Femenino } \\
\text { Total }\end{array}$ & $\begin{array}{c}84(58) \\
60(42) \\
144(100)\end{array}$ & $\begin{array}{l}474(55,4) \\
382(44,6) \\
856(100)\end{array}$ & $\begin{array}{l}558(55,8) \\
442(44,2) \\
1000(100)\end{array}$ \\
\hline \multicolumn{4}{|l|}{ IMC } \\
\hline $\begin{array}{l}<25 \\
25-30 \\
>30 \\
\text { Total }\end{array}$ & $\begin{array}{c}12(8,3) \\
60(41,7) \\
72(50) \\
144(100)\end{array}$ & $\begin{array}{c}436(50,9) \\
367(42,9) \\
53(6,2) \\
856(100)\end{array}$ & $\begin{array}{c}448(44,8) \\
427(42,7) \\
125(12,5) \\
1000(100)\end{array}$ \\
\hline \multicolumn{4}{|l|}{ Perímetro abdominal } \\
\hline $\begin{array}{l}\text { M: }<90 \mathrm{H}:<94 \\
\text { M: }>90 \mathrm{H}:>94 \\
\text { Total }\end{array}$ & $\begin{array}{l}26(18,1) \\
118(81,9) \\
144(100) \\
\end{array}$ & $\begin{array}{l}718(83,9) \\
138(16,1) \\
856(100)\end{array}$ & $\begin{array}{l}744(74,4) \\
256(25,6) \\
1000(100)\end{array}$ \\
\hline \multicolumn{4}{|c|}{ Ejercicio 30 minutos diarios } \\
\hline $\begin{array}{l}\text { Sí } \\
\text { No } \\
\text { Total }\end{array}$ & $\begin{array}{c}29(20,1) \\
115(79,9) \\
144(100)\end{array}$ & $\begin{array}{l}237(27,3) \\
619(72,3) \\
856(100)\end{array}$ & $\begin{array}{l}266(26,6) \\
734(73,4) \\
1000(100)\end{array}$ \\
\hline \multicolumn{4}{|l|}{ Frutas/verduras } \\
\hline $\begin{array}{l}\text { Todos los días } \\
\text { No todos los días } \\
\text { Total }\end{array}$ & $\begin{array}{l}48(33,3) \\
96(66,7) \\
144(100)\end{array}$ & $\begin{array}{l}423(49,4) \\
433(50,6) \\
856(100)\end{array}$ & $\begin{array}{l}471(47,1) \\
529(52,9) \\
1000(100)\end{array}$ \\
\hline \multicolumn{4}{|l|}{ Medicación para HTA } \\
\hline $\begin{array}{l}\text { Sí } \\
\text { No } \\
\text { Total }\end{array}$ & $\begin{array}{l}57(39,6) \\
87(60,4) \\
144(100) \\
\end{array}$ & $\begin{array}{c}75(8,8) \\
781(91,2) \\
856(100) \\
\end{array}$ & $\begin{array}{l}132(13,2) \\
868(86,8) \\
1000(100) \\
\end{array}$ \\
\hline \multicolumn{4}{|c|}{ Valores previos de glucemia alta } \\
\hline $\begin{array}{l}\text { Sí } \\
\text { No } \\
\text { Total }\end{array}$ & \begin{tabular}{|c|}
$53(36,8)$ \\
$91(63,2)$ \\
$144(100)$ \\
\end{tabular} & $\begin{array}{c}23(2,7) \\
833(97,3) \\
856(100)\end{array}$ & $\begin{array}{c}76(7,6) \\
924(92,4) \\
1000(100)\end{array}$ \\
\hline \multicolumn{4}{|c|}{ Historia familiar de diabetes } \\
\hline $\begin{array}{l}\text { Sí: 1er o 2do grado } \\
\text { No } \\
\text { Total }\end{array}$ & \begin{tabular}{|c|}
$119(82,6)$ \\
$25(17,4)$ \\
$144(100)$ \\
\end{tabular} & $\begin{array}{l}338(39,5) \\
518(60,5) \\
856(100)\end{array}$ & $\begin{array}{l}457(45,7) \\
543(54,3) \\
1000(100) \\
\end{array}$ \\
\hline \multicolumn{4}{|l|}{ Nivel educativo } \\
\hline $\begin{array}{l}\text { Ninguno } \\
\text { Escuela incompleta } \\
\text { Escuela completa } \\
\text { Educación superior } \\
\text { Total }\end{array}$ & $\begin{array}{c}1(0,7) \\
45(31,3) \\
51(35,4) \\
47(32,6) \\
144(100)\end{array}$ & $\begin{array}{c}6(0,7) \\
171(20) \\
337(39,4) \\
342(40) \\
856(100)\end{array}$ & $\begin{array}{c}7(0,7) \\
216(21,6) \\
388(38,8) \\
389(38,9) \\
1000(100)\end{array}$ \\
\hline \multicolumn{4}{|l|}{ Estrato } \\
\hline $\begin{array}{l}1 \text { y } 2 \\
3 \text { y } 4 \\
5 \text { y } 6 \\
\text { Total }\end{array}$ & $\begin{array}{c}49(34) \\
83(57,6) \\
12(8,3) \\
144(100)\end{array}$ & $\begin{array}{c}289(33,8) \\
485(56,7) \\
82(9,6) \\
856(100)\end{array}$ & $\begin{array}{c}338(33,8) \\
568(56,8) \\
94(9,4) \\
1000(100)\end{array}$ \\
\hline \multicolumn{4}{|l|}{ Régimen de salud } \\
\hline $\begin{array}{l}\text { Subsidiado } \\
\text { Contributivo } \\
\text { Total }\end{array}$ & $\begin{array}{l}24(16,7) \\
120(83,3) \\
144(100)\end{array}$ & $\begin{array}{l}155(18,1) \\
701(81,9) \\
856(100)\end{array}$ & $\begin{array}{l}179(17,9) \\
821(82,1) \\
1000(100)\end{array}$ \\
\hline
\end{tabular}


Tabla 3. Resultados de las glucemias en ayunas obtenidos de los pacientes que se realizaron los estudios

\begin{tabular}{l|c|c|c}
\hline & $\begin{array}{c}\text { Mujeres n } \\
\text { (\% grupo) } \\
\text { (\% total) }\end{array}$ & $\begin{array}{c}\text { Hombres } \mathbf{n} \\
\text { (\% grupo) } \\
\text { (\% total) }\end{array}$ & $\begin{array}{c}\text { Total } \mathbf{~} \\
\text { (\% total) }\end{array}$ \\
\multicolumn{4}{l}{ Glucemia en ayunas (mg/dl) } \\
\hline$<\mathbf{1 0 0}$ & $7(58,3)(29,2)$ & $6(50)(25)$ & $13(54,2)$ \\
$\mathbf{1 0 0 - 1 2 5 , 9}$ & $5(41,7)(20,8)$ & $4(33,3)(16,7)$ & $9(37,5)$ \\
$\mathbf{2 1 2 6}$ & $0(0)(0)$ & $2(16,7)(8,3)$ & $2(8,3)$ \\
Total & $12(100)(50)$ & $12(100)(50)$ & $24(100)$ \\
\hline
\end{tabular}

es similar a lo encontrado en los estudios de validación FINDRISC en Colombia por Arbeláez-Gómez y cols. ${ }^{(15)}$, y Barengo y cols. ${ }^{(7)}$, donde la prevalencia de sobrepeso y obesidad fue de más del $50 \%$. En el estudio DEMOJUAN ${ }^{(17)}$ se encontró que el $82 \%$ de hombres y mujeres con puntaje entre 13 y 20 tenía sobrepeso y obesidad, y aproximadamente el 49,3\% de quienes tuvieron un puntaje menor de 13 tenía un peso normal que dista ligeramente de lo descrito en el presente estudio, principalmente por mayor porcentaje de participantes hallados en sobrepeso/obesidad (91,7\%).

En el estudio ColDRISC de Barengo y cols., que validó la herramienta ajustando los puntos de corte de perímetro abdominal para Colombia $(94 \mathrm{~cm}$ para hombres y $90 \mathrm{~cm}$ para mujeres), encontró una prevalencia general del 46,2\% para obesidad abdominal, lo que contrasta con el 57,5\% encontrado en DEMOJUAN, ya que este último usó criterios de la OMS (102 cm para hombres y $88 \mathrm{~cm}$ para mujeres) los cuales, en Colombia, sobreestimaban el porcentaje de mujeres con obesidad abdominal y subestimaba el de hombres ${ }^{(7,17)}$. La prevalencia de obesidad abdominal que se encontró en este estudio fue aún menor siendo del 25,6\% pero, con relación al puntaje, tuvo el comportamiento esperado, encontrando un perímetro abdominal aumentado en el $81,9 \%$ de quienes obtuvieron un puntaje de riesgo y normal en el 83,9\% de quienes no lo obtuvieron.

En Venezuela, en un estudio realizado en 2014 por Paredes y cols. en donde se aplicó el cuestionario FINDRISC se concluyó que el $41,34 \%$ de la población se encontraba en sobrepeso y el $19,8 \%$ en obesidad, $62,6 \%$ reportaron sedentarismo y $38,3 \%$ no tenían dieta balanceada, resultados similares a los descritos en Manizales ${ }^{(19)}$.

En México en 2017, González-Pedraza y cols., evaluaron pacientes con una media de edad similar $(49,9 \pm 15,1)$ pero con una marcada diferencia de géneros $(25,6 \%$ hombres y $74,4 \%$ mujeres) y se encontró que $35,2 \%$ de los pacientes estaban en sobrepeso y $42,4 \%$ en obesidad, además, $26,4 \%$ tenían HTA, lo que contrasta con los hallazgos reportados, pues en este se encontró una mayor prevalencia de sobrepeso pero una marcada menor prevalencia de obesidad y de HTA; más del $60 \%$ de sus participantes obtuvieron puntaje por encima de 13 , comparado con $14,4 \%$ en el presente estudio ${ }^{(20)}$.
En el estudio de Jølle A. y cols. ${ }^{(21)}$, que incluyó 47.694 personas, se encontró una prevalencia de FINDRISC elevado de $11 \%$, ligeramente inferior a la descrita $(14,4 \%)$. Sin embargo, con respecto a los grupos según el sexo, en el estudio de la población noruega se encontró mayor prevalencia en mujeres $(12,1 \%)$ que en hombres $(9,6 \%)$, contrario a este estudio en el cual al distribuir la prevalencia entre hombres y mujeres fue mayor en los hombres.

En cuanto al estilo de vida saludable, este fue un componente débil para los grupos con y sin riesgo en Manizales, resultados similares a los de Ku y Kegels y cols., en el cual se concluyó que las preguntas sobre dieta y ejercicio no contribuyeron significativamente al puntaje total en el FINDRISC ${ }^{(22)}$.

Para la población manizaleña, los factores de riesgo de más aporte en la herramienta se relacionan con el IMC y perímetro abdominal, factores que son modificables y que parece que impactan de manera importante en el riesgo de diabetes. La historia familiar de diabetes se halló en una cantidad importante de participantes en alto riesgo, por lo que es otro elemento a individualizar en la atención médica, se evidenció que cerca de la mitad de la población adulta de Manizales tiene este factor de riesgo que, al ser tan importante y no corregible, refuerza la recomendación de educar en cuanto a los factores modificables para prevenir la aparición de la enfermedad. La clase social predominante en la población fue la clase media y la mayoría de la población tiene educación secundaria completa y educación superior con cifras casi idénticas entre uno y otro grupo. La falta de atención a las recomendaciones brindadas a los pacientes de riesgo se vio reflejada en que no acudieron a ampliar los estudios al respecto, sin poder establecer alguna diferencia por nivel educativo; se debe crear conciencia en la población sobre la DM2 y los factores de riesgo.

Las diferencias encontradas con los otros estudios podrían responder, entre otras cosas, a las diferencias de las muestras encontradas, por ejemplo una amplia población femenina mientras que este estudio encontró una distribución de la población más homogénea, también las diferencias culturales con la población de los estudios extranjeros podrían explicar los hallazgos disímiles.

Por último, dentro de las fortalezas de este estudio se debe mencionar que es un estudio pionero exploratorio en utilizar la herramienta FINDRISC para describir el riesgo de diabetes en una población (como lo recomienda la guía de práctica colombiana de DM2), lo cual puede ser útil para identificar la población en riesgo y crear conciencia. La principal debilidad del estudio es la forma en la que se llevó a cabo el muestreo; sin embargo, al ser un estudio exploratorio, en una región geográficamente quebrada, limitaba la sistematización y aleatorización de la misma, pero al ser una ciudad intermedia, los sitios en los que se tomó la muestra ofrecían puntos de encuentro común para obtener homogeneidad en la muestra, como se logró. 


\section{Agradecimientos}

A la institución I.P.S. Médicos Internistas de Caldas por el aporte académico y de insumos para la evaluación física y las glucometrías, así como la calibración de los equipos.

\section{Declaración de fuentes de financiación y posibles conflic- tos de interés}

La institución I.P.S. Médicos Internistas de Caldas realizó el aporte de insumos para la evaluación física y las glucometrías, así como la calibración de los equipos.

Los autores declaran no tener conflictos de interés.

\section{Referencias}

1. Cefalu W T. Standards of medical care in diabetes-2017. Diabetes care. 2017; 40 (1): s1-135.

2. Tuomilehto J, Lindström J, Eriksson JG, Valle TT, Hämäläinen H, IlanneParikka P, et al. Prevention of type 2 diabetes mellitus by changes in lifestyle among subjects with impaired glucose tolerance. N Engl J Med. 2001; 344 (18): 1343-50.

3. Molina DI. Diabetes: Epidemiología, fisiopatología, diagnóstico, clínica, cambios terapéuticos en el estilo de vida y tratamiento farmacológico. $1^{\circ}$ ed. Manizales: Gráficas Guzmán; 2012.

4. Tuomilehto J. Cardiovascular risk: prevention and treatment of the metabolic syndrome. Diabetes Res Clin Pract. 2005; 68 (2): s28-s35.

5. Rosolova H, Pelikanova T, Motovska Z. ESC guidelines on diabetes, pre-diabetes, and cardiovascular diseases developed in collaboration with EASD. Summary of the document prepared by the Czech Society of Cardiology. Cor et Vasa. 2014; 56 (2): e190-e205.

6. International Diabetes Federation. Diabetes Atlas, 7th edition. Brussels (Belgium): International Diabetes Federation; 2015.

7. Barengo NC, Tamayo DC, Tono T, Tuomilehto J. A Colombian diabetes risk score for detecting undiagnosed diabetes and impaired glucose regulation. Primary Care Diabetes. 2017; 11 (1): 86-93.

8. Aschner PM, Muñoz OM, Girón D, García OM, Fernández-Ávila D, Casas LA et al. Guía de práctica clínica para la prevención, diagnóstico, tratamiento y seguimiento de la diabetes mellitus tipo 2 en la población mayor de 18 años. Colombia Médica. 2016; 47 (2): 109-31.

9. Ministerio de Salud y Protección Social. Análisis de situación de salud Colombia 2014. Vol 1. 1o ed. Bogotá: Imprenta Nacional de Colombia; 2014.

10. Rodríguez J, Ruiz F, Peñaloza E, Eslava J, Gómez LC, Sánchez H, et al. Encuesta Nacional de Salud 2007. Departamento Caldas. 1ํed. Bogotá: Fundación Cultural Javeriana de Artes Gráficas -JAVEGRAF-; 2009.

12. Kengne AP, Beulens JW, Peelen LM, Moons KG, van der Schouw YT, Schulze $\mathrm{MB}$, et al. Non-invasive risk scores for prediction of type 2 diabetes (EPIC-InterAct): a validation of existing models. Lancet Diabetes Endocrinol. 2014; 2 (1): 19-29.

13. Waugh NR, Shyangdan D, Taylor-Phillips S, Suri G, Hall B. Screening for type 2 diabetes: a short report for the National Screening Committee. Health Technol Assess 2013; 17(35): 1-90.

14. Kahn R, Alperin P, Eddy D, Borch-Johnsen K, Buse J, Feigelman J, et al. Age at initiation and frequency of screening to detect type 2 diabetes: a cost effectiveness analysis. Lancet 2010; 375 (9723): 1365-74.

15. Tankova T, Chakarova N, Atanassova I, Dakovska L. Evaluation of the Finnish Diabetes Risk Score as a screening tool for impaired fasting glucose, im paired glucose tolerance and undetected diabetes. Diabetes Res Clin Pract. 2011; 92 (1): 46-52.

16. Gómez-Arbeláez D, Alvarado-Jurado L, Ayala-Castillo M, Forero-Naranjo L, Camacho PA, López-Jaramillo P. Evaluation of the Finnish Diabetes Risk Score to predict type 2 diabetes mellitus in a Colombian population: A longitudinal observational study. World J Diabetes. 2015; 6 (17): 1337-44.

17. Lindström J, Ilanne-Parikka P, Peltonen M, Aunola S, Eriksson JG, Hemiö K, et al. Sustained reduction in the incidence of type 2 diabetes by lifestyle intervention: follow-up of the Finnish Diabetes Prevention Study. Lancet. 2006; 368 (9548): 1673-9.

18. Barengo NC, Acosta T, Arrieta A, Ricaurte C, Mayor D, Tuomilehto JO. Screen ing for people with glucose metabolism disorders within the framework of the DEMOJUAN Project (DEMOnstration area for primary prevention of type 2 diabetes, JUAN Mina and Barranquilla, Colombia). Diabetes Metab Res Rev. 2013; doi: 10. 1002/dmrr.2462.

19. López Jaramillo P, Calderón C, Castillo J, Escobar ID, Melgarejo E, Parra GA Prediabetes in Colombia: Expert Consensus. Colomb Med (Cali). 2017; 48 (4): 191-203.

20. Paredes N, Alejandría Ojeda M, López J, López A, Rosales J, Scaglia R, et al. Aplicación del test FINDRISC para cálculo del riesgo de padecer diabetes mellitus tipo 2. Med Interna (Caracas). 2014; 30 (1): 34-41

21. González Pedraza A, Ponce Rosas ER, Toro Bellot F, Acevedo Giles O, Dávila Mendoza R. Cuestionario FINDRISC FINnish Diabetes Risk Score para la detección de diabetes no diagnosticada y prediabetes. Archivos en medicina familiar. 2018; 20 (1): 5-13.

22. Jølle A, Midthjell K, Holmen J, Tuomilehto J, Carlsen SM, Shaw J, et al. Impact of sex and age on the performance of FINDRISC: the HUNT Study in Norway. BMJ Open Diabetes Research and Care. 2016; 4 (1): e000217.

23. Ku GM, Kegels G. The performance of the Finnish Diabetes Risk Score, a modified Finnish Diabetes Risk Score and a simplified Finnish Diabetes Risk Score in community -based cross-sectional screening of undiagnosed type 2 diabetes in the Philippines. Prim Care Diabetes. 2013; 7 (4); 249-59. 